\title{
Reinforcing behaviours other than abstinence was effective in reducing drug use
}

\author{
Iguchi MY, Belding MA, Morral AR, et al. Reinforcing operants other than abstinence in drug abuse treatment: an effective \\ alternative for reducing drug use.J Consult Clin Psychol 1997 Jun;65:421-8.
}

\section{Objective}

To compare the effectiveness of 3 reinforcement strategies (standard treatment, reinforcement based on urinanalysis samples, and reinforcement based on a treatment plan) in reducing drug use.

\section{Design \\ Randomised controlled trial with 24 weeks of follow up.}

\section{Setting}

Methadone maintenance treatment clinic in New Jersey, USA.

\section{Patients}

103 patients (mean age $36 \mathrm{y}, 63 \%$ men) who were admitted to a methadone maintenance treatment clinic for opiate dependence between June 1993 and August 1994. Exclusion criteria were a positive test for methadone, methadone treatment within the previous month, inability to attend treatment or testing sessions, or psychotic symptoms.

\section{Intervention}

35 patients received standard treatment of regular individual counselling sessions with opportunities to earn the privilege of take home medication. 27 patients received urinalysis based reinforcement in which patients earned 10 vouchers for each drug free urine specimen. Bonus vouchers for the first drug free specimen were offered to patients who tested positively for drugs. 41 patients received treatment plan based reinforcement in which patients could earn 30 vouchers each week for completing specific tasks with objective criteria which were defined in the previous week by counsellors and patients. Vouchers were worth US $\$ 0.50$.

\section{Main outcome measure}

Number of drug free urine samples across 4 time periods: 1-6 weeks (baseline), 7-12 weeks (intervention), 13-18 weeks (intervention), and 19-24 weeks (follow up).

\section{Main results}

Analysis was by intention to treat. Abstinence was increased as a function of time and treatment $(\mathrm{p}<0.05)$. Patients who received the treatment plan based reinforcement showed an increase in the number of drug free urine samples over time $(\mathrm{p}<0.05)$ (table)

\section{Conclusion}

A reinforcement strategy based on a treatment plan devised by patients and counsellors was more effective in reducing drug use than standard treatment or reinforcement of abstinence.

Mean \% of drug free urine samples

\begin{tabular}{lllll}
\hline \multicolumn{5}{l}{ Time period $(w k)$} \\
\hline Treatment groups & $1-6$ & $7-12$ & $13-18$ & $19-24$ \\
Treatment plan & 9 & 21 & 29 & 34 \\
Urinanalysis & 15 & 16 & 18 & 24 \\
Standard treatment & 21 & 24 & 17 & 20 \\
\hline
\end{tabular}

Source of funding: National Institute on Drug Abuse.

For article reprint:Dr M Y Iguchi, Drug Policy Research Center, RAND, 1700 Main Street, PO Box 2138, Santa Monica, CA 90407-2138, USA. Fax +1 3103934818

\section{Commentary}

Patients seeking treatment for drug problems are a heterogeneous group. One of the central challenges to the treatment of drug problems is providing treatments that address this heterogeneity of patient characteristics and problems. This study by Iguchi $e t$ al shows and evaluates how this can be done within a study of 3 reinforcement conditions.

In the condition of most interest, patients earned money vouchers for meeting treatment goals that were identified each week on an individualised basis in consultation with programme counsellors (treatment plan condition). An issue of specific interest is that the targets within the treatment plan condition were deliberately set to be achievable so that all participants would have an opportunity to receive positive reinforcement. A disadvantage of previous contingency schemes has often been that many clients failed to earn even a single reinforcer. ${ }^{1}$ Also, the individualised treatment plan condition included targets other than abstinence. The results suggest that this approach to treatment offers some benefits over a standard treatment package (counselling plus take home privileges) and over a condition in which reinforcement was conditional upon providing "clean" urine samples. Only the patients who were allocated to the individualised contingency condition showed decreased use of illicit drugs.

The interpretation of these results is not entirely straightforward because of the many differences between the treatment plan and the urinalysis based conditions. Not least of these is the complicated (and often unacknowledged) issue of the perceptions, attitudes, and expectations of patients in response to the experimental procedures. The treatment of drug problems should not be seen as merely a technical matter. For instance, in what ways is allocation to a treatment condition affected by awareness of other conditions? Could patients in the urinalysis condition have felt "cheated" because their own goals were more difficult to achieve than those for the treatment plan patients? These are fascinating and important issues, and it is to the considerable credit of the authors that they have acknowledged and found space to discuss these ambiguities.

Michael Gossop, $\mathrm{PhD}$ National Addiction Centre Maudsley Hospital London, UK

1 Stitzer ML, Iguchi MY, Felch LJ. Contingent take-home incentive: effects on drug use of methadone maintenance patients. Clin Psychol 1992;60:927-34. 